\title{
Polimorfismo del gen de la banda 3 eritrocítica en grupos étnicos de Costa Rica
}

\author{
M. Chaves-Villalobos ${ }^{1}$, G. Jiménez-Arce ${ }^{1} \&$ M. Sandí-Díaz ${ }^{1,2}$ \\ 1 Centro de Investigación en Hematología y Trastornos Afines (CIHATA), Universidad de Costa Rica. \\ 2 Departamento de Genética Médica y Metabolismo, Hospital Nacional de Niños "Dr. Carlos Sáenz Herrera" Centro de \\ Especialidades Médicas. Correspondencia: marioch@cariari.ucr.ac.cr, fax (506) 223-1385.
}

Recibido 26-VII-2002. Corregido 12-X-2003. Aceptado 24-XI-2003.

\begin{abstract}
Polymorphism of the erythrocyte band 3 gene (EPB3) in ethnic groups of Costa Rica. Band 3 (AE1) is one of the most abundant proteins in the membrane of the human erythrocyte. This protein works as an anionic $\mathrm{CI}$ - and HCO3- exchanger and it also functions as an anchor for several proteins of the erythrocyte's cytoesqueleton. Several mutations have been described and many polymorphic variants have been associated to hereditary spherocytosis. The identification of a genetic marker at position 5' of the AE1 gene could be associated to several molecular defects of the erythrocyte. This genetic marker is a restriction fragment length polymorphism (RFLP) produced by restriction enzime Pst I. For this polymorphism a total of 216 individuals belonging to seven different populations were analyzed: one from the Central Valley, two African descendants (Limón and Guanacaste) and four Amerindians (Bribri, Cabecar, Maleku and Guaymi). The most frequent allele in the Amerindian population was no 1. No significant differences were found with respect to the Hardy-Weinberg equilibrium in six of the populations, although the Guaymi group does present significative differences. Rev. Biol. Trop. 52(3): 659-663. Epub 2004 Dic 15.
\end{abstract}

Key Words: Band 3, ethnic groups, erythrocyte, Pst I, polymorphism, Costa Rica.

Palabras clave: Banda 3, grupos étnicos, eritrocito, Pst I, polimorfismo, Costa Rica.

La proteína banda 3 eritrocítica (AE1) es una de las proteínas transmembrana mejor estudiada en las células de mamíferos, debido principalmente a su papel en el transporte de $\mathrm{CO}_{2}$ de los tejidos a la sangre y a la descarga del $\mathrm{CO}_{2}$ de la sangre a los pulmones, ayudando así a prevenir cambios en el $\mathrm{pH}$ del citosol (Berne et al. 1993), además por su abundancia en el eritrocito humano donde se presenta en $1.2 \mathrm{x}$ $10^{6}$ copias por eritrocito (Delaunay 1995).

$\mathrm{Su}$ función es el intercambio aniónico cloruro / bicarbonato (AE1), presenta un peso molecular entre los 90000 y 100000 daltons (Jay et al. 1986) y se expresa principalmente en eritrocitos; no obstante se ha determinado la presencia de AE1 en otras células como las $\alpha$-intercalares de los túbulos distales del riñón (Bruce et al. 1998) y en las neuronas (Marguerite et al. 1991).
La proteína banda 3 esta conformada por 911 aminoácidos distribuidos en tres segmentos proteicos, el primero de ellos es un dominio transmembranal (posición 404-883) constituido por 14 segmentos responsables del intercambio cloruro-bicarbonato en la célula; los otros dos segmentos son dominios citoplasmáticos en los extremos amino y carboxilo terminales (posición 1-403 y 884-911, respectivamente), estos son sitios de anclaje de varias proteínas del citoesqueleto como la $\alpha$-espectrina, $\beta$-espectrina, ankyrina, la hemoglobina y las proteínas denominadas banda 4.1 y 4.2 (Delaunay 1995). Por lo que mutaciones en dichos sitios se han asociado a trastornos del eritrocito, como esferocitosis hereditaria y ovalocitosis (Palek 1990).

El gen AE1 fue mapeado en la región cromosómica 17q21-qter (Lux et al. 1989, Showe 
et al. 1987), presenta un tamaño de $17 \mathrm{~kb}$ y está constituido por 20 exones. Las características más importantes del gen son la carencia de cajas TATA y CCAAT en el promotor y una gran variedad de secuencias consenso de factores de transcripción (Delaunay 1995). Se han descrito muchas mutaciones que incluyen codones de terminación prematura o codones de terminación que son el resultado de deleciones e inserciones que producen ARNm inestables o bandas 3 truncadas que no pueden plegarse correctamente en la membrana celular; otros tipos de mutaciones son sustituciones de un aminoácido en posiciones conservadas o deleciones dentro de una cadena de aminoácidos conservados (Maillet et al. 1995). Muchas variantes se han descrito dentro del gen de la banda 3 entre ellas la Memphis (Yannoukakos et al. 1991, Jarolim et al. 1992), Tuscaloosa (Jarolim et al. 1992) y el polimorfismo Pst I (Jenkins et al. 1993).

Entre los exones 2 y 3 se halla una región de $1.5 \mathrm{~kb}$ en el extremo 5 ' del gen, esta región contiene un fragmento BamH1 de $1.1 \mathrm{~kb}$ que incluye el polimorfismo Pst 1 (Jenkins et al. 1993). Estudios de este polimorfismo en grupos humanos muestran frecuencias alélicas para poblaciones de ancestría europea de 0.8 para el alelo 1 y 0.2 para el alelo 2 , y para individuos de ancestría africana de 0.46 (alelo 1) y 0.54 (alelo 2) (Jenkins et al. 1993).

En otros estudios, Stewart et al. (1989) demostraron que existe ligamiento entre el polimorfismo Pst I de la banda 3 y el gene que codifica para el receptor del factor de crecimiento neuronal. Presentando frecuencias para los alelos 1 y 2 de 0.78 y 0.22 , respectivamente, para la población europea y de 0.7 y 0.3 para pigmeos de Zaire.

El objetivo de esta investigación es conocer la distribución del polimorfismo Pst I en varios grupos étnicos de Costa Rica.

\section{MATERIAL Y MÉTODOS}

Con el propósito de analizar el polimorfismo Pst 1 del gene de la banda 3 del eritrocito, se analizaron 216 individuos de siete poblaciones del banco de ADN que posee el CIHATA de la Universidad de Costa Rica, correspondientes a los tres grupos étnicos que componen la población costarricense. Dos grupos con ancestría africana, uno de la Costa Atlántica $(n=30)$ y otro de la Costa Pacífica ( $\mathrm{n}=31$ ) los cuales fueron clasificados fenotipicamente como afrodescendientes; la población hispanomestiza está representada por individuos del Valle Central $(n=31)$, las otras cuatro poblaciones fueron grupos amerindios bribri $(\mathrm{n}=31)$, cabecar $(n=31)$, guaymí $(n=31)$ de la zona Sur, y maleku de Guatuso $(\mathrm{n}=31)$ de la zona Norte de Costa Rica.

La extracción de ADN se hizo a partir de sangre periférica tomada con EDTA-K ${ }_{3}$, siguiendo el método de cloruro de sodio de Miller et al. (1988). La amplificación del ADN y el análisis de restricción de PstI se hizo de acuerdo a Jenkins (1993), el cual permite la identificación de los alelos, el primero produce una banda de $1.375 \mathrm{~kb}$; el segundo alelo produce dos bandas una de $0.975 \mathrm{~kb}$ y una de $0.4 \mathrm{~kb}$.

Se calcularon las frecuencias alélicas y genotípicas de estos dos alelos por el método de conteo de genes. Se realizó una prueba exacta usando una cadena de Markov, con un nivel de confianza del 95\% y 1 grado de libertad, como prueba para el equilibrio de HardyWeinberg (HW) en cada una de las poblaciones y un Análisis de Varianza Molecular (AMOVA) para diferencias entre y dentro grupos y poblaciones.

\section{RESULTADOS}

Las frecuencias fenotípicas y alélicas encontradas se presentan en el Cuadro 1. En este, se aprecia que las frecuencias genotípicas son muy similares entre los tres grupos étnicos; encontrándose predominancia de los heterocigotas $(1,2)$, como es de esperar en una población de ancestría trihíbrida (Morera y Barrantes 1995). Sin embargo, en los hispanomestizos del Valle Central el genotipo $(1,1)$ se encuentra en menor frecuencia $(10 \%)$, con respecto a los amerindios y afrodescendientes 
CUADRO 1

Frecuencias fenotípicas y alélicas del polimorfismo del gen Banda 3 en poblaciones étnicas de Costa Rica

\begin{tabular}{|c|c|c|c|c|c|c|}
\hline \multirow[t]{3}{*}{ Población } & \multirow{2}{*}{\multicolumn{3}{|c|}{$\begin{array}{l}\text { Frecuencias } \\
\text { Genotípicas }\end{array}$}} & \multirow{3}{*}{$\begin{array}{c}\text { Muestra } \\
\text { (n) }\end{array}$} & \multicolumn{2}{|c|}{ Frecuencias Alélicas } \\
\hline & & & & & & \\
\hline & 1,1 & 1,2 & 2,2 & & $\mathrm{p}(1)$ & $\mathrm{q}(2)$ \\
\hline \multicolumn{7}{|l|}{ Hispanomestizos } \\
\hline Valle Central & 3 & 21 & 7 & 31 & 0.4355 & 0.5645 \\
\hline \multicolumn{7}{|l|}{ Amerindios } \\
\hline Bribri & 14 & 16 & 1 & 31 & 0.7097 & 0.2921 \\
\hline Cabecar & 12 & 17 & 1 & 30 & 0.6833 & 0.3167 \\
\hline Guaymí & 8 & 22 & 1 & 31 & 0.6129 & 0.3871 \\
\hline Maleku & 9 & 13 & 9 & 31 & 0.5000 & 0.5000 \\
\hline \multicolumn{7}{|l|}{ Afrodescendientes } \\
\hline Limón & 15 & 12 & 4 & 31 & 0.6774 & 0.3226 \\
\hline Guanacaste & 10 & 19 & 2 & 31 & 0.6290 & 0.3710 \\
\hline Costa Rica (todos) & 71 & 120 & 25 & 216 & 0.6065 & 0.3935 \\
\hline
\end{tabular}

(*) en desequilibrio de $\mathrm{H}-\mathrm{W}$

donde solo es superado por el genotipo $(1,2)$. En este sentido se observa que al utilizar un AMOVA la diferencia entre grupos no es significativa $(\mathrm{p}=0.19)$ con un porcentaje de variación de $2.22 \%$. La variación entre poblaciones dentro de grupos es de $1.28 \%$ y la variación poblacional es de $96.5 \%$.

Con relación a las dos poblaciones de ancestría africana, se observa que las frecuencias genotípicas de Limón presentan una proporción mayor de individuos homocigotas para el genotipo $1.1(48.4 \%)$ y un $38,7 \%$ para los heterocigotas. Sin embargo, Guanacaste presenta un $61.3 \%$ de individuos heterocigotas, y un $32.2 \%$ para los homocigotas del alelos 1 ; en esta población los homocigotas 2,2 representan el $6 \%$ de los individuos. Se observa que no existen diferencias significativas para el equilibrio de Hardy-Weinberg en estas dos poblaciones (Limón $\mathrm{p}=0.68$ y Guanacaste $\mathrm{p}=0.14$ ).

Como se aprecia en el Cuadro 1, la población costarricense del Valle Central, clasificada como hispanomestiza muestra mayor frecuencia del alelo 2. Se nota un predominio de individuos heterocigotas $(67.7 \%)$ y una disminución considerable de los individuos homocigotas para el alelo 1 que representan el $9.5 \%$. No hay diferencias significativas para el equilibrio de Hardy-Weinberg en esta población $(\mathrm{p}=0.07)$.

En las poblaciones de ancestría amerindia se encuentra en promedio frecuencias de 0.62 para el alelo 1 y de 0.38 para el alelo 2 . Como se observa, esta población presenta frecuencias genotípicas bajas para los homocigotas 2,2 con respecto a la población del Valle Central.

Así la población bribrí presenta una frecuencia genotípica de individuos heterocigotas de $51.6 \%$, con casi un $70 \%$ de homocigotas para el alelo 1. Según la prueba exacta sí hay equilibrio HW $(p=0.38)$.

Con relación a las frecuencias genotípicas de la población cabecar, esta población presenta un predominio de individuos heterocigotas $(57 \%)$ y homocigotas $1,1(40 \%)$, manteniéndose en equilibro HW ( $\mathrm{p}=0.20)$.

La población maleku de Guatuso presenta la mayor frecuencia de individuos para el alelo 2 de entre los cuatro grupos amerindios analizados. Estando en igual proporción de individuos para el alelo 1. Al analizar las frecuencias genotípicas, se observa que se mantiene la relación. La población se encuentra en equilibrio de HW ( $\mathrm{p}=0.5)$.

En la población guaymí, los individuos heterocigotas se encuentran en mayor 
proporción (70.97\%) en comparación con los otros cuatro grupos amerindios. Sin embargo, los homocigotas 1,1 y 2,2 presentan una reducción considerable $(25.8 \%$ y $3 \%$ respectivamente) con respecto a las otras poblaciones. De acuerdo a la prueba exacta en esta población hay diferencias significativas por lo que no se ajusta al equilibrio de HW ( $p=0.01$ ).

\section{DISCUSIÓN}

La acción de factores evolutivos, históricos, demográficos y de cruzamiento originan la presencia o ausencia de ciertos alelos en las poblaciones humanas. En este sentido la población costarricense en general, se caracteriza por ser una mezcla trihíbrida, compuesta en su mayoría de genes de origen europeo, amerindio y en menor grado de origen africano de acuerdo a la zona de procedencia (Morera et al. 2001, 2003). Con relación a las frecuencias alélicas, la población del Valle Central muestran una mayor proporción de individuos con el alelo 2 que los otros grupos étnicos estudiados; este resultado demuestra que habrá una mayor proporción de individuos heterocigotas y homocigotas para el alelo 2 en el Valle Central, como un reflejo a la mayor cantidad de individuos con una clara ancestría europea en esta región, ya que los resultados concuerdan con los obtenidos por Jenkins et al. (1993) en poblaciones europeas. El Valle Central presenta la mayor densidad de individuos por $\mathrm{km}^{2}$ de Costa Rica, favoreciendo con ello, una mayor presencia de cruces al azar dentro de la población; proporcionando así el aumento de individuos heterocigotas para ciertos alelos dentro de la población.

La población afrocostarricense es el resultado de dos procesos migratorios que ocurrieron en épocas diferentes, una a mediados de la Colonia donde gran cantidad de africanos llegaron a Guanacaste y la otra ocurrió a finales del siglo pasado con la llegada de personas de Jamaica y de otras poblaciones afro-derivadas del Caribe para la construcción del ferrocarril al Atlántico costarricense (Sáenz et al. 1971,
1974, 1986). Las frecuencias alélicas de estos dos grupos son muy parecidas entre sí y a las informadas por Stewart et al. (1989) en una población de Zaire en donde hay predominancia del alelo 1. Sin embargo, hay diferencias en las frecuencias genotípicas, especialmente en las frecuencias de heterocigotas siendo mayor en la población afrodescendiente de Guanacaste, esto posiblemente por el asentamiento y cruce con poblaciones de otros orígenes, como chinos y anglosajones que podrían estar originando un cambio en la dinámica de esa población. Con relación a los grupos amerindios se observa que existe heterogeneidad genética en todos los grupos. Sin embargo, el grupo guaymí presenta un aumento significativo de heterocigotos que produce el desvió de la población en relación con el equilibrio de Hardy-Weinberg. Estudios posteriores en demografía genética de está población, podrían reflejar realmente que factores evolutivos influyen en el grupo guaymí. Los cuatro grupos amerindios estudiados presentan diferencias genotípicas en cuanto al número de individuos homocigotas para el alelo 2, habiendo predominio del alelo 1, especialmente entre los grupos del sur del país (bribrí, cabecar, guaymí) y el grupo del norte de Costa Rica (maleku de Guatuso) el cual presenta equilibrio en la distribución en sus frecuencias. Estas diferencias podrían deberse a un mayor número de cruces consanguíneos entre los grupos del sur en contraposición a los cruces azarosos en los malekus y a la mezcla de los grupos amerindios del sur con otros grupos étnicos de Costa Rica.

\section{RESUMEN}

Banda 3 (AE1) es una de las proteínas más abundantes de la membrana del eritrocito humano. Ésta funciona como un intercambiador aniónico cloruro / bicarbonato y es el punto de anclaje de varias proteínas del citoesqueleto del eritrocito. Se han descrito varias mutaciones y muchas variantes polimórficas se han asociado a esferocitosis hereditaria. La identificación de un marcador genético en el extremo 5' del gene AE1 podría asociarse a varios defectos moleculares del eritrocito. Este marcador genético es un fragmento de restricción de longitud polimórfica "RFLP" producido por la endonucleasa de restricción Pst 
I. Se analizaron para este polimorfismo un total de 216 individuos de siete poblaciones: una hispanomestiza (Valle Central), dos afrodescendientes (Limón y Guanacaste) y cuatro amerindias (bribri, cabecar, maleku y guaymí). El alelo más frecuente en la población hispanomestiza fue el 2 , mientras en los grupos afrodescendientes y amerindios se encontró el 1. No se observaron diferencias significativas con respecto al equilibrio de Hardy-Weinberg en seis de las poblaciones, sin embargo, el grupo Guaymí si presenta diferencias significativas con respecto al equilibrio de Hardy-Weinberg.

\section{REFERENCIAS}

Berne, R.M. \& M.N. Levy. 1993. Physiology. Mosby-Year Book, St Louis, Missouri. U.S.A. pp. 20-21

Bruce, L.J., R. Unwin, O. Wrong \& M. Tanner. 1998. The association between familial distal renal tubular acidosis and mutations in the red cell anion exchanger (band 3, AE1) gene. Biochem. Cell. Biol. 76: 723-728.

Delaunay, J. 1995. Genetic disorders of the red cell membrane. Critical Reviews in Oncology/Hematology 19: 79-110.

Jarolim, P., J. Palek, H.L. Rubin, J.T. Prchal, C. Korsgren \& C.M. Cohen. 1992. Band 3 Tuscaloosa: Pro327ÆArg 327. Substitution in the Cytoplasmic Domain of Erythrocyte Band 3 Protein Associated with spherocytic Hemolytic Anemia and PartialDeficiency of Protein 4.2. Blood 80: 523-529.

Jarolim, P., H.L. Rubin \& S. Zhai. 1992. Band 3 Memphis: A Widespread polimorphism with abnormal electrophoretic mobility of erythrocyte Band 3 protein caused by substitution AAGÆGAG (Lys ÆGlu) in codon 56. Blood 80: 1592-1598.

Jay, L. 1986. Structural aspects of the Red Cell Anion Exchange Protein. Ann. Rev. Biochem. 55: 511-538.

Jenkins, P.B., P.G. Gaallagher \& B.G. Forget. 1993. Analysis of a Pst I polymorphism of the human erythrocyte band 3 gene (EPB3). Br. J. Haem. 85: 816-818.

Lux, S.E., K.M. John, R. Kopito \& H. Lodish. 1989. Cloning and characterization of Band 3, the human erythrocyte anion-exchange protein (AE1). Proc. Nath. Acad. Sci. USA. 86: 9093.

Mailet, P., A. Valier \& W.H. Reinhart. 1995. Band 3 Chur: and variant associated with band 3- deficient hereditary spherocytososis and substitution in a highly con- served position of transmembrane segment 11. Br. J. Haem. 91: 804-810.

Marguerite, M., B. Kay, J. Hughes, I. Zagon \& Lin. 1991. Brain Membrane protein Band 3 performs the same Functions as Erithrocyte Band 3. Cell 88: 27782782 .

Miller, S.A., D. Dykes \& H.F. Polesky. 1988. A simple salting out procedure for extracting DNA from human nucleated cell. Nucleic Acid Res. 16: 1215.

Morera, B. \& R. Barrantes. 1995. Genes e historia: el mestizaje en Costa Rica. Revista de Historia 32: 43-64.

Morera, B., R. Marín-Rojas \& R. Barrantes. 2001. Análisis de varios marcadores genéticos clásicos en la población de Costa Rica. Rev. Biol. Trop. 49: 1237-1252.

Morera, B., R. Marín-Rojas \& R. Barrantes. 2003. Gene Admixture in the Costa Rican Population. Ann. Hum. Genet. 67: 71-80.

Palek, J. \& S. Lambert. 1990. Genetics of the Red Cells Membrane Skeleton. Sem. Hematology 27: 290-332

Sáenz, G.F., G. Arroyo, J. Jiménez, A. Gutiérrez, M. Barrenechea, E. Brilla \& E. Valenciano. 1971. Investigación de hemoglobinas anormales en la población de raza negra costarricense. Rev. Biol. Trop. 19: 251-260.

Sáenz, G.F., G. Arroyo, M.A. Alvarado, G. Montero, J. Jiménez \& E. Valenciano. 1974. Hemoglobinas anormales en una población estudiantil universitaria. Rev. Biol. Trop. 21: 417-424.

Sáenz, G., M. Cháves \& E.M. Quintana. 1986. Las hemoglobinopatias en Costa Rica aspectos históricos, culturales y epidemiológicos. Rev. Cost. Cien. Med. 7: 95-106.

Showe, L.C., M. Ballantine \& K. Huebner. 1987. Localization of the gene for the erythroid exchage protein, Band 3 (EPB3) to the chromosome 17. Genomics 1: 71-76.

Stewart, E.A., R. Kopito \& A.M. Bowcock. 1989. A Pst I polymorphism for the erythrocyte surface protein band 3 (EPB3) to human Chromosome 17. Genomics 1: 71-76.

Yannoukakos, D., C. Vasseur \& C. Driancourt. 1991. Human Erithrocyte Band 3 polymorphism (Band 3 Memphis): Characterization of the Structural modification. (Lys 56ÆGlu ) by Protein Chemistry methods. Blood 78: 1117-1120. 